\title{
Applications of the Psion organiser at Strathclyde University Library
}

\author{
Dennis Nicholson, Systems Officer, Strathclyde University Library
}

\section{The organiser and library applications}

\subsection{Introduction}

The Psion organiser is a small hand-sized computer about the size of a (thick) pocket diary. Despite its size, it is a complete computer by any reasonable definition, with processor, 16-32Kbyte memory, operating system, programming language, resident software, and the ability to communicate with peripherals such as printers and light pens, as well as with other microcomputers. The resident programs are an electronic diary, a personal database system, a calculator, a clock, an alarm facility and a programming facility. The programming facility allows programs to be created, edited, saved, compiled and inserted into the main menu in such a way that they may be run with a single keystroke. At time of writing (May 1988), the basic machine comes in two models. Each has the facilities and capabilities described above, but they vary in the on-board memory available. The 16Kbyte model costs approximately $£ 100$, the $32 \mathrm{Kbyte}$ model costs approximately $£ 130$. Enhancements available include:

- blank 'datapaks' which can increase the data-storage capacity to $288 \mathrm{Kbyte}$;

- a communications link, allowing amongst other things, the upload and download of files to or from another microcomputer, and the dumping of data and programs to a (serial) printer;

- software packages, including financial programs, a spreadsheet, word processing.

The organiser and its peripherals are easy to use and, for the most part, 'userfriendly'. Datapaks are simple to use, and to fit and remove, changing batteries is a simple matter, as is the process of linking to peripherals. Manuals are clear and helpful and the organiser is easily customised to personal (or organisational) requirements. One criticism relates to the size (approximately $2 \frac{1}{2}$ " by 3 ") and layout (not QWERTY) of the keyboard. However, use of this improves with practice and the handy size of the machine compensates for the initial awkwardness. The screen is, of necessity, small but, generally speaking, adequate. A short program, listed in the manual, can be typed in to permit control of access by password. It should be noted that, being very portable, the organiser is easy to steal.

\subsection{Assessment of in-built facilities}

\section{a) Calculator}

This is relatively sophisticated and would probably do anything a librarian or information scientist is likely to need in the course of his or her duties. A useful feature is the ability to post-edit calculations. Ten memory stores are available and their contents can be passed to external programs, a very useful feature.

\section{b) Personal database facility}

This is extremely useful if swift access is required to a wide range of difficultto- remember information. Telephone numbers, names and addresses and other similar data can be easily saved. Retrieval is possible by typing any four consecutive characters from the record (e.g. DENNIS could be retrieved using DENN, ENNI, NNIS). The Systems Unit at Strathclyde University Library has found this facility invaluable for storing information about library systems - such as all the parameters of all the terminals on the Geac System. The Unit has begun to compile a shared 'systems' database available to each of the systems staff through the exchange of datapaks. 


\section{c) Diary facility}

This allows diary entries to be made in half-hour slots. Each entry can have an alarm sound on request up to 59 minutes before the time of the entry. The diary can be searched by date and by subject. It can be saved to a datapak and reloaded, tidied, erased, and listed consecutively. Surprisingly, there is no MOVE (to another time slot) facility. The entry to be moved has to be re-typed. Nevertheless, this facility can be extremely helpful in organising busy and complex schedules.

\subsection{Facilities available through programming}

The organiser can be programmed using a language called OPL, a fact which widens its potential applications greatly. This has led to the availability of a growing number of commercially-available software packages. For example, at least one supplier of library systems (Geac) has adapted the organiser for use as a portable data-capture unit. However, it also offers the individual user a means of extending and adapting the organiser. The simple program for calculating percentages, listed in Figure 1 to give a 'flavour' of OPL, is an example of this, as is the statistics logging and reporting system described later.

\section{Statistics logging on the organiser}

\subsection{The statistics}

The Systems Unit at Strathclyde University Library comprises two members of professional staff, each of whom is required to record statistics on how their time is spent, recording for each non-trivial task carried out:

- general category (R\&D or operational systems);

- total time spent on the task;

- total time spent using a computer;

- system or developing system worked on;

- description of the work carried out;

- date.

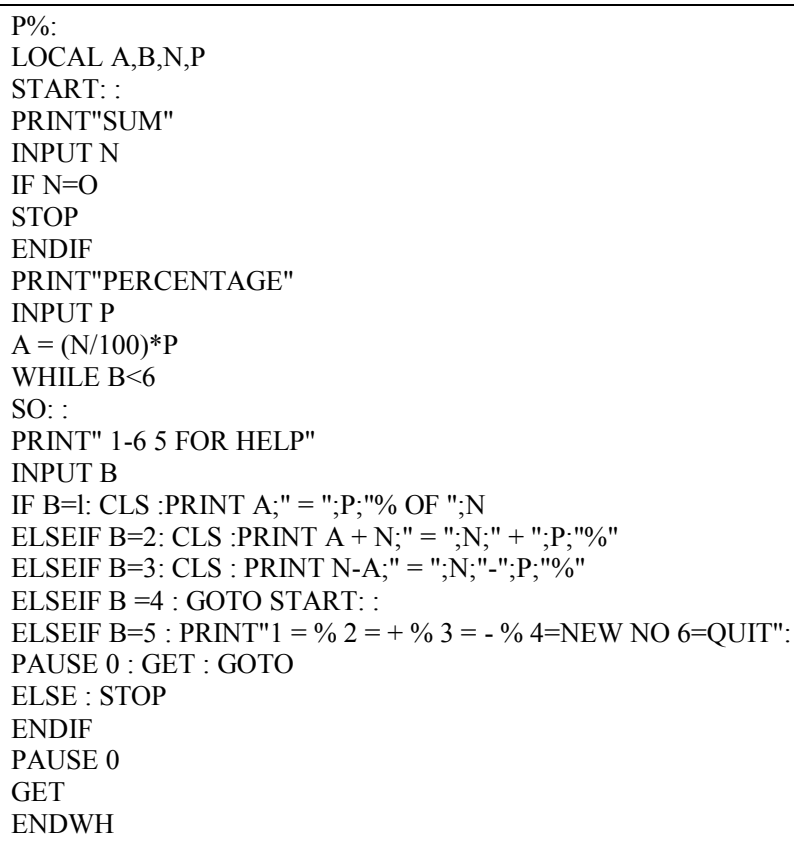

Figure 1. An OPL program for calculating percentages 
The Unit is required to combine, structure, and summarise the data recorded in monthly reports to the University Librarian. Prior to January 1988, the recording and reporting process was manual. The raw data were recorded by making rough, unstructured, and sometimes illegible entries in Unit diaries. Monthly report production was a tedious task involving the deciphering, structuring, summarising, and typing up of these sometimes incomplete data. This made it an unpopular and time-consuming task which tended to ensure that reports were produced several weeks late at best and were less complete in detail than they might have been.

\subsection{The automated process: an overview}

The automated alternative to the system described above was put into live use in January 1988. In the new system, the two members of Unit staff log their statistics on individual organisers, using an inhouse program called WSTAT (for Write STATistics). Since this program has been inserted in the organiser's main menu, and has an initial letter unique within that menu, invoking the logging program is a simple matter of pressing the ' $\mathrm{W}$ ' key. Once this is pressed, the user is prompted to enter:

- $\quad \mathrm{N}$ or $\mathrm{O}$ for new/operational system;

- a three-letter code identifying the system itself;

- the computer time in minutes;

- the total time in minutes;

- a short description of the work carried out.

The date is taken from the organiser's calendar and added automatically, and the resulting record structured from the first, and legible - is written to a file. At the end of the month, the files from the two organisers are downloaded to an IBM PS2/50, using the Psion communications package, and the files are subsequently cleared down ready for next month's log. On the microcomputer, the files are combined and processed using a dBase HI plus command file, which then produces the kind of structured monthly report illustrated in Figure 2. Using this new system, the monthly statistical report, which previously took several hours to produce, and was typically several weeks late in production, may be produced in less than 20 minutes on the first working day of the new month.

\subsection{The organiser logging programs}

\section{a) WSTAT}

WSTAT is the main logging program. Having pressed W (for WSTAT) on the organiser's main menu the user is presented with the options:

Xit, Con, Date:

- if ' $\mathrm{X}$ ' is chosen the program exits to the main menu of the organiser without recording anything.

- if ' $\mathrm{D}$ ' date is chosen the user is prompted to enter a date other than the current day's date.

- if ' $\mathrm{C}$ ' is chosen, the user is prompted with: $N, O=N e w, O p . S y s$.

and must enter either ' $\mathrm{N}$ ', if the work being logged relates to a new or developing system, or ' $\mathrm{O}$ ', if the work being logged relates to an operational system.

The user is then prompted with:

SYSTEM:

in order that the three-letter code identifying the system in question may be entered. If EXE is pressed at this point (the Psion equivalent of a return key) a facility to search for and display, or to display 
sequentially, the currently available codes is invoked. Exiting from this facility brings back the prompt SYSTEM:

$\begin{array}{rlrr}\text { Day System } & \text { Comp } & \text { Over } & \text { Description } \\ \text { of Name } & \text { uter } & \text { all of task } \\ \text { Mth } & \text { time } & \text { time } & \end{array}$

** CATEGORY: OPERATIONAL SYSTEMS

I5 AQ: BK ORDERS CDROM

2 AQ: GENERAL

14 CA: GEAC CATALOGUING

14 CA: GEAC CATALOGUING

7 CA: GEAC CATALOGUING

9 CA: GEAC CATALOGUING

9 CA: GEAC CATALOGUING

9 CA: OCLC ONLINE LINK

8 CA: OCLC ONLINE LINK

2 R\&I: ONLINE SERVICE

7 R\&I: ONLINE SERVICE

14 R\&I: ONLINE SERVICE

7 R\&I: ONLINE SERVICE

2 R\&I: ONLINE BILLS

9 R\&I: ONLINE BILLS

7 R\&I: PRESTEL

14 R\&I: JANET CATALOGUES

15 R\&I: DOMESDAY PROJ.

8 R\&I: CD ROM

7 R\&I: CD ROM

8 RS: CIRCULATION SYS.

9 RS: CIRCULATION SYS.

2 RS: CIRCULATION SYS.

7 RS: TELEX/E.MAIL SYS

14 RS: TELEX/E. MAIL SYS

9 RS: TELEX/E. MAIL SYS

2 SY: STATISTICS PROG

1 SY: STATISTICS PROG

9 SY: PSION

7 SY: SYS.UN. INVOICES

8 SY: SYS.UN. INVOICES

14 SY: SYS.UN. INVOICES

8 SY: GENERAL

3 SY: GENERAL

3 SY: GENERAL

2 SY: GENERAL

9 SY: GENERAL

2 SY: GENERAL

9 SY: GENERAL

9 SY: GENERAL

J0 SY: GENERAL

9 SY: GENERAL

11 SY: GENERAL

11 SY: GENERAL

I SY: GENERAL

1 SY: GENERAL

14 SY: GENERAL
90.0090 .00 BRS SOFTWARE/BOOKBANK

$0.0030 .00 \quad$ GEAC DEMIO ETC

60.0090 .00 PRINT/ANALYSE * KEEP

60.0060 .00 LOOK INTO MPDUPL ERROR

60.0060 .00 GRSNS FOR DUPLICATE RECORDS

60.0090 .00 MPDUPL PROJECT

90.0090 .00 INVESTIGATE MBOVRN PROBLEM

30.0060 .00 CHECK NEW TERMINAL DOWNLOAD

$30.00 \quad 30.00 \quad$ TERMINAL PARAMIETERS

$15.00 \quad 15.00$ COLLECTORS CHECK

120.00120 .00 ONLINE TRAINING/AH

$30.00 \quad 60.00$ OLD IBM SEARCH STRATEGIES

$30.0030 .00 \quad$ CHECK/ERASE COLLECTORS

$30.00 \quad 30.00$ BILLS PROGRAM UPDATE

0.0015 .00 VAT IN ONLINE BILLING

60.0060 .00 AMSTRAD PRESTEL PROCEDURES

$0.00 \quad 30.00$ AMSTRAD PRESTEL ACCESS

$120.00 \quad 320.00$ ECODISC/INTERACTIVE VIDEO

60.0060 .00 ORGANISE CDROM FILES

0.0075 .00 CDROM SUBS/R+I

$30.00 \quad 30.00$ HUGO SYSTEM ERRORS

30.0060 .00 RESOLVE MUDDLED ITEM TRANSACTION

$30.00 \quad 30.00$ CIRC TABLES CHANGES

60.0060 .00 MONITOR/TIDY TGOLD FILES

30.0030 .00 REPLY TO TGOLD MESSAGES

60.0060 .00 TGOLD FILES

$30.00 \quad 60.00$ PAPER TO ID MTNG

60.0060 .00 MONTHLY STATS ETC

0.0030 .00 CHASE FORMATTER ORDER WITH CSS

0.0060 .00 CHECK + CLEAR INVOICES

$0.00 \quad 45.00$ GEAC INVOICE PROGRESS

$0.00 \quad 30.00$ HITACHI/ARCHETYPE INVOICE

$0.00 \quad 30.00 \quad$ O.ALLEN $/ 9000$ COURSE

$30.00 \quad 30.00$ WORDPROCESSOR TRAINING

$30.00 \quad 30.00$ MAIL AND EMAIL

15.0015 .00 SLOW OVERNIGHTS PROBLEM

60.0090 .00 WORD PROCESSOR TRAINING/MR

$10.00 \quad 15.00$ TAPE OF **OCLZ

0.0030 .00 NEW 9000 LINE/MARSHALLS

15.0015 .00 DUMP FEC CHECK TO PRINTER

30.0030 .00 CHECK SYSTEM/OVERNIGHTS ETC

0.00420 .00 GEAC SOFTWARE

DEMO/EDINBURGH

$30.00 \quad 30.00$ CHECK SYSTEMS UP

0.00420 .00 GEAC SOFTWARE

DEMO/EDINBURGH

60.0060 .00 GEAC OVERNIGHTS PROBLEM

$30.00 \quad 30.00$ TGOLD EMAIL WORK

0.0030 .00 GEAC/DATAPROJECTOR DEMO

Figure 2. Systems Unit statistical report - a sample report 


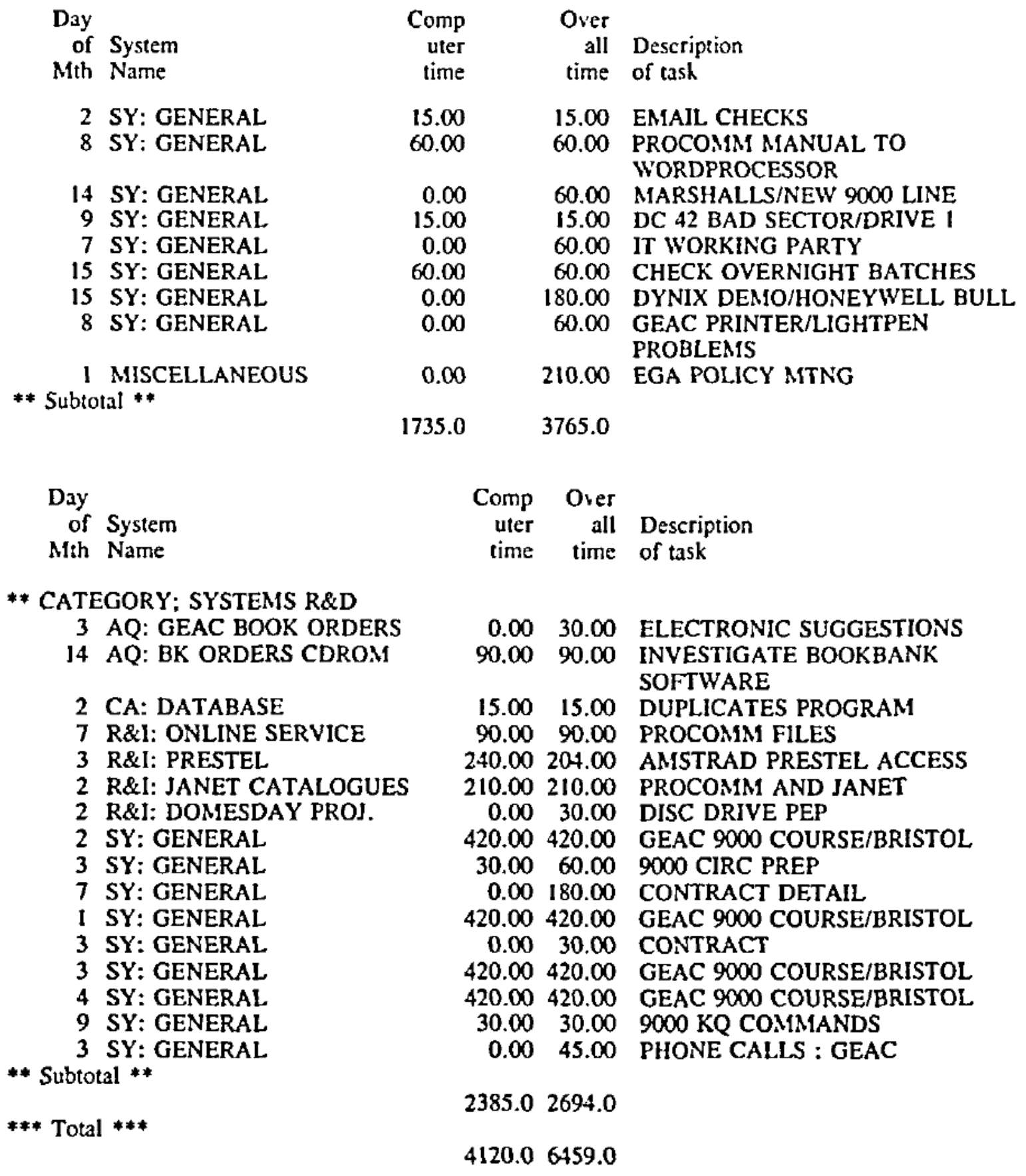

Figure 2 (cont.). Systems Unit statistical report - a sample report

Once the appropriate system code has been entered, the user is prompted with:

- COMPUTER TIME: (the time spent using a computer, to be entered in minutes)

- OVERALL TIME: (the total time working on the system in question, again to be entered in minutes)

Entering the overall time produces the prompt:

DESCRIPTION:

in order that the kind of work done on the system in question may be recorded. Entering this, the last part of the log entry, produces the prompt: 


\section{$X t, C n$, Date,Ed,Q:}

Entering ' $\mathrm{X}$ ', for exit, returns control to the organiser's main menu, having written the record just entered to the statistics file. Entering ' $\mathrm{C}$ ', for continue, allows a second entry to be recorded from the "N,O = New, Op.Sys." prompt. Entering ' $\mathrm{D}$ ' is equivalent to ' $\mathrm{C}$ ', except that the user may enter the date to be recorded against the next entry, in preference to having the system date recorded. Entering 'E', for edit, allows the entry just recorded to be edited before it is written to the file. Entering 'Q', for quit, returns control to the organiser's main menu without writing the record just entered to the statistics file.

\section{b) XSTAT}

The second program, XSTAT, has two functions. Function one allows the file of statistical entries to be listed, a useful feature if the user needs confirmation that an entry has been made or made correctly. Function two, used only at the beginning of a new month when the statistics file has been downloaded, allows the file to be cleared down ready for a new set of entries.

\section{c) $Z C O D E$}

The third program, ZCODE, has one function: it allows new three-letter statistical codes to be added to the list of system codes.

\subsection{Downloading the statistics to a PS2/50 and dBase III Plus}

When all of the statistics for a given month have been logged, the files from the two organisers are downloaded to two separate files on an IBM PS2/50 microcomputer, using the Psion communications link. The communications link package comprises a communications cable, communications software for the organiser, and communications software for an IBM compatible microcomputer. It will connect the organiser to 'a printer, desktop computer, modem, or any-device which has an RS232 or RS423 port'. Amongst other things, it will enable the printing of files and programs from the organiser, the use of the organiser as a terminal (e.g. for use with telex or electronic mail services), or, as in this case, file transfer between the organiser and an IBM compatible microcomputer.

The file transfer process takes only a few minutes and involves connecting each organiser in turn to the microcomputer's serial port, invoking the software on both the organiser and the microcomputer, and choosing the 'transmit' option on the organiser's communications menu (the parameters for communication having been previously set up and saved). In this instance, Psion's own protocol is used to control the communications process (other alternatives available are xmodem and no protocol). Once the two files have been transferred to the microcomputer, they are cleared down on the organisers, so that the new month's statistics can be recorded.

\subsection{Processing the files and producing the report}

Once the files are on the microcomputer, the process of producing the report is virtually automatic. A dBase III Plus program file is run which converts the files to dBase format, combines them into one file, translates the category codes and system codes into the headings seen in the report, indexes and sorts the resulting files, and outputs it in the structured report format shown in Figure 2.

The two organiser files are converted to dBase HI Plus format using the dBase APPEND FROM (filename) DELIMITED WITH, command, the Psion field separator (TAB) having been globally replaced with a comma during the file transfer process (DELIMITED WITH "TAB" does not appear to work in dBase). Appending each file to the same dBase file produces a single file of Unit statistics. This is then put in system code order using the dBase SORT command, the system codes and the ' $\mathrm{O}$ ' and ' $\mathrm{N}$ ' categories are translated into their full names using the REPLACE command, indexed by category using the INDEX command and printed out in the format shown in Figure 2 using the 
REPORT FORM command. Provided that there are no new system codes to add before processing can take place, the whole process is automatically handled by a single command file and takes less than 10 minutes. If new codes are required, the process takes a little longer, perhaps another 10 minutes.

\section{Concluding remarks}

The organiser has proved a significant aid in a number of areas of Systems Unit work and there are now plans to investigate its potential in the wider area of library operations as a whole. The possibility of extending its use in the gathering and reporting of library statistics is now being actively investigated, the hope being that it will both reduce the workload of staff in this area and significantly increase the speed with which monthly statistical reports are produced. There is also some interest in the potential usefulness of having a large body of those library statistics (currently held manually) in machine readable form for the first time. Another area of interest is in the development of databases of common interest which can be created and updated centrally on a microcomputer and downloaded regularly to individual organisers. This idea has yet to be discussed in full. However, a small experimental step towards it has already been taken, the library's internal telephone directory having been put on a microcomputer so that it may be updated and downloaded to organisers in this manner.

Dennis Nicholson is Systems Officer at Strathclyde University Library, 101 St. James' Road, Glasgow G41 0NS 